\title{
HIGH RESOLUTION OBSERVATIONS OF THE MAGNETIC
}

\section{FIELD IN IC 342}

\author{
MARITA KRAUSE \\ Max-Planck-Institut für Radioastronomie, Auf dem Hügel 69, W-5300 Bonn 1, FRG
}

December 10, 1992

\section{Introduction}

The face-on spiral galaxy IC 342 is known to possess an axisymmetric spiral magnetic field (ASS) with magnetic field lines being orientated inwards everywhere at least in the analyzed radial range between 5 and $13 \mathrm{kpc}^{1}$ (Krause et al., 1989a; Sokoloff et al., 1992). The ASS field configuration seems to be even with respect to the galactic plane.

The polarized emission itself, however, is pronounced along long and thin spiralshaped ridges in the above mentioned radial range, especially in the eastern half of the galaxy, as observed at $\lambda 20 \mathrm{~cm}, \mathrm{D}$-array, with the VLA with an angular resolution of $45^{\prime \prime}$ (Krause et al., 1989a). These ridges are generally located outside the optical spiral arm region, but one of them crosses the south-eastern spiral arm near its bifurcation. At this crossing point (located at $\alpha_{50}=3^{\mathrm{h}} 42^{\mathrm{m}} 30^{\mathrm{s}}, \delta_{50}=67^{\circ} 52^{\prime}$ ) the linear polarization has its maximum at $\lambda 20 \mathrm{~cm}$, whereas the total intensity peaks along the central north-western spiral arm (around $\alpha_{50}=3^{\mathrm{h}} 41^{\mathrm{m}} 30^{\mathrm{s}}, \delta_{50}=67^{\circ} 54^{\prime}$ ). In order to analyze the uniform magnetic field pattern and depolarization effects also in the inner part of IC 342 we observed this region at $\lambda 6 \mathrm{~cm}$ with higher resolution.

\section{Observations}

Observations of the total intensity and linear polarization were performed with the VLA of the National Radio Astronomy Observatory ${ }^{2}$ in its D-configuration. As the primary beam of the single VLA telescopes at $\lambda 6 \mathrm{~cm}$ does not cover the whole area of interest we had two observation runs with different phase centres, one about 3.5 north-west of the nucleus, the other about $7^{\prime}$ south-east of the nucleus, for 12 hours each. Both data sets were reduced separately using the standard AIPS package. An estimated zero-spacing flux was used in both areas in total intensity in order to correct for the negative bowls. The data were tapered to an angular resolution of $16^{\prime \prime} \mathrm{HPBW}$ and $45^{\prime \prime}$ HPBW each. The r.m.s. noise of the maps with $16^{\prime \prime} \mathrm{HPBW}$ is $25 \mu \mathrm{Jy} / \mathrm{b}$.a. in total power and $15 \mu \mathrm{Jy} / \mathrm{b}$.a. in polarized intensity, whereas the $45^{\prime \prime}$ resolution maps have an r.m.s. noise of $40 \mu \mathrm{Jy} / \mathrm{b}$.a. in total and $20 \mu \mathrm{Jy} / \mathrm{b} . \mathrm{a}$. in polarized emission. The data sets of each area were corrected for

${ }^{1}$ For consistency's sake with our previous papers we will assume a distance of $3.1 \mathrm{Mpc}$, hence $1^{\prime} \triangleq 0.9 \mathrm{kpc}$, even if Turner and Hurt (1992) have good arguments to adopt a distance to IC 342 of only $D=1.8 \mathrm{Mpc}$ which would increase our linear resolution by a factor of 1.7.

2 The NRAO is operated by Associated Universities, Inc., under contract with the National Science Foundation 
primary beam attenuation and clipped outside $70 \%$ of the primary beam area. Finally the corresponding data sets of both areas were combined to single maps, respectively. At least in total power large-scale structure is missing. So, the values of the degree of linear polarization $p$ may be overestimated. However, comparison with $\lambda 6 \mathrm{~cm}$ observations made with the 100-m telescope in Effelsberg (Gräve and Beck, 1988) at even lower resolution show similar values for $p$.

\section{Results}

The resulting maps of the linearly polarized intensity at $\lambda 6 \mathrm{~cm}$ are given in Figs. 1 and 2 as contour plots for $45^{\prime \prime}$ HPBW and 16" HPBW angular resolution, respectively. The circular full lines correspond to $70 \%$ of the primary beam area at $\lambda 6 \mathrm{~cm}$. All data points outside these regions were clipped. The position angles of the vectors give the orientation of the observed E-vectors in Fig. 1 whereas they are rotated by $90^{\circ}$ in Fig. 2. Their length is in both figures proportional to the estimated degree of linear polarization.

When comparing these and the corresponding total power maps they exhibit some peculiar differences in the distribution of the radiation: At $\lambda 6 \mathrm{~cm}, 45^{\prime \prime} \mathrm{HPBW}$, the total emission is stronger in the north-western than in the south-eastern region, similar to the $\lambda 20 \mathrm{~cm}, 45^{\prime \prime}$ HPBW map (Krause et al., 1989a). This is also valid for the polarized intensity at $\lambda 6 \mathrm{~cm}$ (see Fig. 1), whereas the polarized intensity at $\lambda 20 \mathrm{~cm}$ shows a significant maximum in the south-eastern area (Krause et al., 1989a). The changes in the general distribution of the radiation are even more dramatic in the $\lambda 6 \mathrm{~cm}, 16^{\prime \prime} \mathrm{HPBW}$, maps: There total power as well as linear polarization are stronger in the north-western area of IC 342 than in the southeastern one (see Fig. 2). These differences in the distribution of linear polarization between maps of different wavelengths (but equal resolution) on one hand and those of different resolution (at the same wavelength) on the other hand indicate dissimilar depolarization effects in the different areas of IC 342. This will be discussed at the end of this paper.

The rotation measure $R M$ could be determined between $\lambda 6 \mathrm{~cm}, 45^{\prime \prime}$ HPBW, and the previous $\lambda 20 \mathrm{~cm}$ observations at equal resolution (Krause et al., 1989a). The values are generally small and range in most parts where they could be determined between $-10 \mathrm{rad} \mathrm{m}^{-2}$ and $10 \mathrm{rad} \mathrm{m}^{-2}$. Hence, the observed E-vectors at $\lambda 6 \mathrm{~cm}$ are not much $\left(\lessgtr 2^{\circ}\right)$ affected by Faraday rotation. Therefore a rotation of the observed vectors by $90^{\circ}$ represents directly the orientation of the uniform magnetic field perpendicular to the line of sight $\left(B_{\perp}\right)$ and is shown in Fig. 2.

The most remarkable features in Fig. 2 can be summarized as follows:

- The polarized intensity is again concentrated along thin spiral-shaped ridges, similar to the ridges at $\lambda 20 \mathrm{~cm}$ in the eastern half of the galaxy further outwards.

- The ridges of polarized emission are located in the interarm region of the optical spiral arms.

- The dominant north-western ridge can be traced inwards up to a distance of only $1 \mathrm{kpc}$ to the nucleus. There are indications of another small ridge even further inwards.

- The uniform magnetic field component $B_{\perp}$ is orientated along the ridges. 
Fig. 1. Contour map of linear polarization at $\lambda 6 \mathrm{~cm}$ with an angular resolution of $45^{\prime \prime}$ HPBW. The orientation of the vectors gives the observed E-field.

Fig. 2. Contour map of linear polarization at $\lambda 6 \mathrm{~cm}$ with $16^{\prime \prime} \mathrm{HPBW}$. The vectors indicate the orientation of the $B$ field.

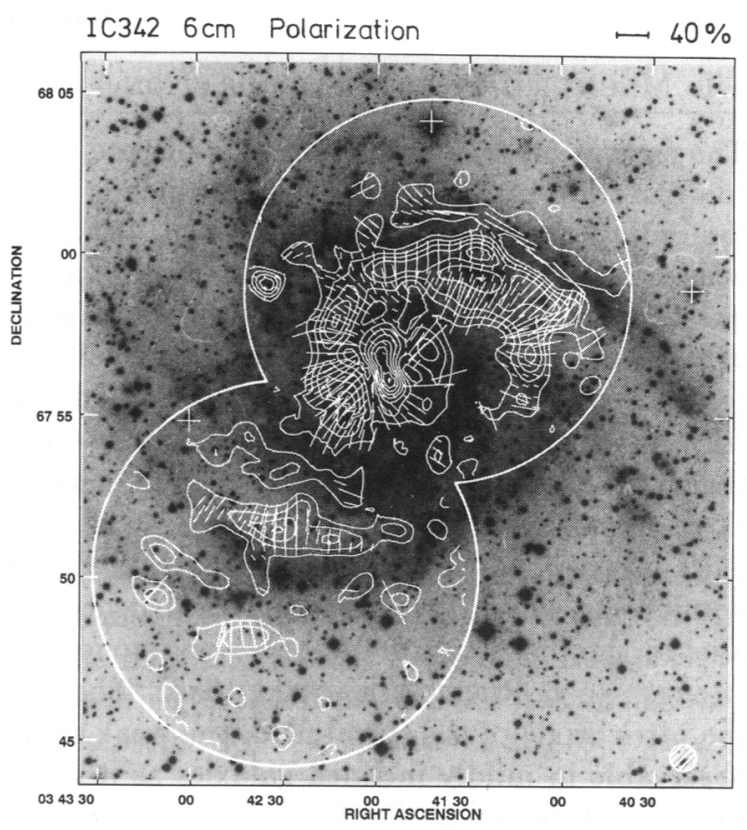

Peak flux $=6.5711 \mathrm{E}-04$ JY/BEAM
Levs $=4.0000 \mathrm{~B}-05+(-4.00,-2.00,2.000$
$4.000,6.000,8.000,10.00,12.00,14.00$

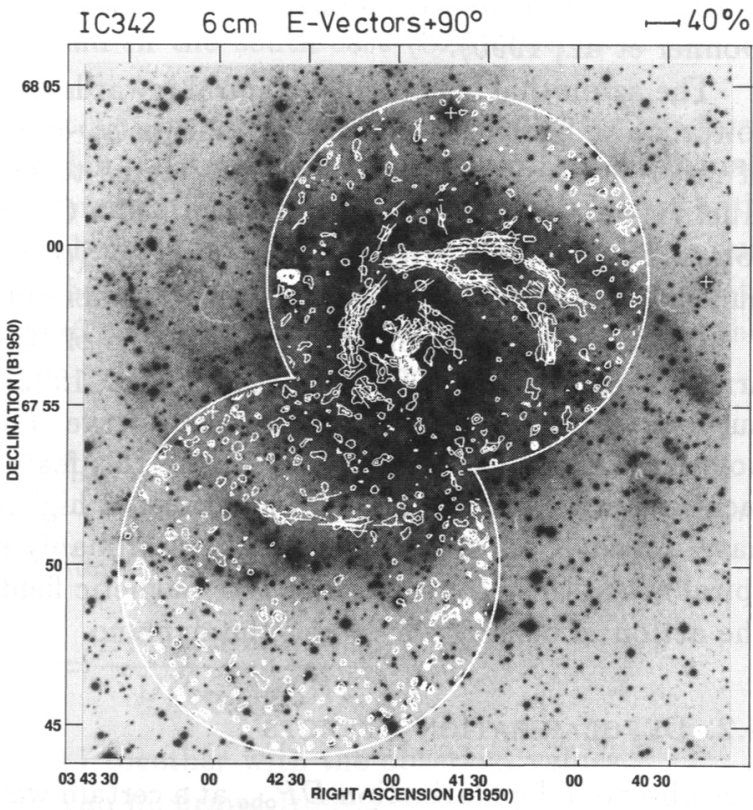

Peak flux $=4.5035 E-04$ JY/BEA

Leys $=1.5000-05 *(2.000 .4 .000,6.000$
$8.000,10.00,12.00,14.00,16.00,18.00$ 


\section{Discussion}

\subsection{Magnetic Field Configuration}

The present observations fit into the picture that IC 342 has an ASS large-scale field configuration in the galactic plane which is shown to spiral inwards everywhere in the radial range between 5 and $13 \mathrm{kpc}$. The magnetic field lines should close somewhere (in the halo). From observational point of view it is a long time outstanding question whether the magnetic field lines leave the disk mainly in the central region of the galaxy, i.e. that there is a strong poloidal magnetic field component in the central region, or whether the azimuthal field is accompanied everywhere in the disk with a weak $B_{\mathbf{z}}$ component. The latter case is assumed implicitly in the dynamo theory as a dynamo transforms locally kinetic energy into magnetic energy in the three-dimensional space.

The assumed $\alpha \omega$-dynamo in the thin disk generates a magnetic field with a dominating azimuthal component $B_{\varphi}$ (hence the field configuration is mainly parallel to the disk) and radial and $z$-components $\left(B_{\mathrm{r}}\right.$ and $\left.B_{\mathrm{z}}\right)$ of about only $1 / 10$ in strength (e.g. Ruzmaikin et al., 1988). The weak $B_{\mathbf{r}}$ component is observable in the pitch angle of the spiral field, the weak $B_{\mathrm{z}}$ component, however, is not directly observable in mildly inclined galaxies as it gives only a very small contribution to the $R M$ of the azimuthal field.

The observed low rotation measures in the central part of IC 342 exclude the existence of a strong poloidal magnetic field there. Hence it favours the picture of a weak $B_{\mathrm{z}}$ component everywhere in the disk which is, however, not observable. This is also in agreement with model calculations of the $\alpha \omega$-dynamo for a thin disk (e.g. Donner et al., 1990).

The azimuthal field seems to spiral inwards up to at least $1 \mathrm{kpc}$ (or even nearer) towards the nucleus. Considering that an $\alpha \omega$-dynamo can only work in the region of differential rotation of a disk this seems to be a surprising result. Turner and Hurt (1992), however, recently determined a CO rotation curve showing that rigid rotation is confined within a $85 \mathrm{pc}$ radius (corrected for $D=3.1 \mathrm{Mpc}$ ) of the nucleus. The rotation curve slowly rises further out, i.e. there is differential rotation.

The molecular gas in the central region of IC 342 takes the form of two spiral arms (Ishizuki et al., 1990; Turner and Hurt, 1992) and are interpreted by the latter authors as due to an inner spiral density wave. The very inner small ridge in linear polarization in Fig. 2 may be related to this feature, nevertheless our observations lack resolution. Polarization observations at higher resolution of the nuclear region have already been carried out and will probably shed more light on these nuclear spiral arms, their connection to the magnetic field and a possible relation between the action of density waves and the dynamo.

\subsection{Depolarization Effects}

The observed depolarization $D P_{\text {obs }}$ at a certain wavelength $\lambda$ and angular resolution $\Theta_{\mathrm{A}}$ is defined as the ratio of the nonthermal degree of linear polarization $p_{\mathrm{n}}$ (i.e. $p$ corrected for the unpolarized thermal contribution) and the theoretical value $p_{\mathrm{i}} \simeq 73 \%$. Relevant depolarization may be caused by the wavelength-independent 


\begin{tabular}{|c|c|c|c|c|c|c|c|c|}
\hline $\begin{array}{l}\lambda \\
{[\mathrm{cm}]}\end{array}$ & $\begin{array}{l}\Theta_{A} \\
{["]}\end{array}$ & $D P_{\text {obs }}$ & $D P_{\text {beam }}$ & $\overline{D P_{\mathrm{r}}}$ & $\begin{array}{l}\sigma_{\mathrm{RM}} \\
{\left[\frac{\mathrm{rad}}{\mathrm{m}^{2}}\right]}\end{array}$ & $\begin{array}{l}\left\langle n_{e}>\right. \\
{\left[\mathrm{cm}^{-3}\right]}\end{array}$ & $\begin{array}{l}B_{\mathrm{r}} \\
{[\mu \mathrm{G}]}\end{array}$ & $\begin{array}{l}d \\
{[\mathrm{pc}]}\end{array}$ \\
\hline \multicolumn{9}{|c|}{ Area NW: } \\
\hline 6 & 16 & 0.23 & & & & & & \\
\hline 6 & 45 & 0.27 & $0.27-0.31$ & & & & & \\
\hline 20 & 45 & 0.04 & $0.27-0.31$ & $0.13-0.15$ & $4000-4500$ & 0.1 & $8 \pm 2$ & $8-14$ \\
\hline \multicolumn{9}{|c|}{ Area SE: } \\
\hline 6 & 16 & 0.27 & & & & & & \\
\hline 6 & 45 & 0.25 & $0.25-0.36$ & & & & & \\
\hline 20 & 45 & 0.21 & $0.25-0.26$ & $0.81-0.84$ & $200-300$ & 0.04 & $6 \pm 2$ & $3-11$ \\
\hline
\end{tabular}

beam depolarization $D P_{\text {beam }}$ and by the wavelength-dependent differential Faraday rotation $D P_{\mathrm{u}}$ (caused by the uniform magnetic field $B_{\mathrm{u}}$ and depending on the internal rotation measure $R M_{\mathrm{i}}$ ) and the internal Faraday dispersion $D P_{\mathrm{r}}$ (due to the random field component $B_{\mathbf{r}}$ and depending on the dispersion of rotation measure $\sigma_{\mathrm{RM}}$ ) (Burn, 1966; for summary and discussion see also Krause et al., 1989a,b and Ehle and Beck, 1993):

$$
D P_{\text {obs }}\left(\lambda, \Theta_{\mathrm{A}}\right)=D P_{\text {beam }} \cdot D P_{\mathrm{r}} \cdot D P_{\mathrm{u}} .
$$

In the following the different polarization effects will be discussed along the main ridges of linear polarization in the north-western region (NW) of IC 342 centred at $\alpha_{50}=3^{\mathrm{h}} 41^{\mathrm{m}} 30^{\mathrm{s}}, \delta_{50}=67^{\circ} 54^{\prime}$ and in the south-east (SE) centred at $\alpha_{50}=$ $3^{\mathrm{h}} 42^{\mathrm{m}} 30^{\mathrm{s}}, \delta_{50}=67^{\circ} 52^{\prime}$.

As the internal rotation measures (i.e. the observed $R M$ corrected for the foreground rotation $R M_{\mathrm{fg}}=-9 \pm 5 \mathrm{rad} \mathrm{m}^{-2}$ (Krause et al., 1989a)) are very small $\left(\leq 5 \mathrm{rad} \mathrm{m}^{-2}\right.$ ) in both areas, the differential Faraday rotation is negligible at both wavelengths. If we assume that the observed depolarization at $\lambda 20 \mathrm{~cm}$ is only due to Faraday dispersion, we get with the corresponding values for $\sigma_{\mathrm{RM}}$ upper limits for the Faraday dispersion $D P_{\mathrm{r}}$ at $\lambda 6 \mathrm{~cm}, \Theta_{\mathrm{A}}=45^{\prime \prime}$. We then can evaluate the beam depolarization at $45^{\prime \prime}$ as

$$
D P_{\text {obs }}\left(6 \mathrm{~cm}, 45^{\prime \prime}\right) \leq D P_{\text {beam }}\left(45^{\prime \prime}\right) \leq \frac{D P_{\text {obs }}\left(6 \mathrm{~cm}, 45^{\prime \prime}\right)}{D P_{\mathrm{r}}(6 \mathrm{~cm})}
$$

Finally, $D P_{\text {beam }}\left(45^{\prime \prime}\right)$ can be used to determine $D P_{r}(20 \mathrm{~cm})$ as

$$
D P_{\mathrm{r}}(20 \mathrm{~cm})=\frac{D P_{\mathrm{obs}}\left(20 \mathrm{~cm}, 45^{\prime \prime}\right)}{D P_{\text {beam }}\left(45^{\prime \prime}\right)}
$$

The results are summarized in Table 1 together with the observed values.

At least two striking conclusions can be drawn from Table 1:

- $D P_{\text {obs }}$ does not vary much between $16^{\prime \prime}$ and $45^{\prime \prime} \mathrm{HPBW}$ at $\lambda 6 \mathrm{~cm}$, indicating the absence of fluctuations of the magnetic field on scales between 240 and 700 pc (or 
140 and $400 \mathrm{pc}$ for $D=1.8 \mathrm{Mpc}$ ). As the beam depolarization itself is, however, significant there must be a turbulent magnetic field on scales smaller than $240 \mathrm{pc}$.

- The Faraday dispersion $D P_{\mathrm{r}}$ is very different in both areas, being the dominant depolarization mechanism in the NW and small in SE. The corresponding values for $\sigma_{\mathrm{RM}}$ is also given in Table 1 .

The dispersion in rotation measure is defined as $\sigma_{\mathrm{RM}}=\left(0.81 n_{\mathrm{e}} B_{\mathrm{r}}\right)^{2} d L$ (Burn, 1966). $n_{\mathrm{e}}$ is the thermal electron density, $d$ is the scale of the fluctuations of $B_{\mathrm{r}}$ and $L$ is the full line of sight through the Faraday effective region of the galaxy. The turbulent magnetic field strength $B_{\mathrm{r}}$ has been estimated from the radio emission at all observations separately using pressure equipartition. $B_{\mathrm{r}}$ is only slightly weaker in SE than in NW. Values for $n_{\mathrm{e}}$ have been estimated from the observed thermal emission of IC 342 (Hummel and Gräve, 1990) adopting a clumping factor $\left\langle n_{\mathrm{e}}^{2}\right\rangle$ $\left./<n_{\mathrm{e}}\right\rangle^{2}=20$ (Manchester and Mebold, 1977) for both areas. We find significantly different values for $\left\langle n_{\mathrm{e}}\right\rangle$ in both areas (see Table 1). For $L$ we adopt $1 \mathrm{kpc}$ as the thermal emission is indeed correlated with the HII region, but has also a more diffuse component (Hummel and Gräve, 1990). With these values and the very different values for $\sigma_{\mathrm{RM}}$ we find surprisingly equal scales for the fluctuations $d$ of $B_{\mathrm{r}}$ for both areas, namely only about $10 \mathrm{pc}$. This is a rather small value when compared to the usually adopted value of $100 \mathrm{pc}$ in the dynamo theory for the turbulence scale length responsible for the $\alpha$-effect. The result of $d \simeq 10 \mathrm{pc}$ for $B_{\mathrm{r}}$ raises the question whether or not also the turbulence scale length is much smaller which then would increase the dynamo numbers $R_{\alpha}$ and $R_{\omega}$ and the diffusion time scale by the corresponding factor.

Finally, we can explain the different distributions of the polarized emission at $\lambda 20 \mathrm{~cm}$ and Figs. 1 and 2: In the SE area both $D P_{\mathrm{u}}$ and $D P_{\mathrm{r}}$ are small, hence there is already strong linear polarization at $\lambda 20 \mathrm{~cm}$, whereas $D P_{\mathrm{r}}$ (mainly due to the higher $\left\langle n_{\mathrm{e}}\right\rangle$ there) depolarizes the emission in the NW area at $\lambda 20 \mathrm{~cm}$. At $\lambda 6 \mathrm{~cm} D P_{\mathrm{r}}$ has a much smaller effect, thus the degree of linear polarization increases in the NW when compared to $\lambda 20 \mathrm{~cm}$, whereas in the SE the radiation decreases according to the spectral index. The difference between Figs. 1 and 2 is only due to the lower sensitivity at the higher resolution which brings the SE area near the detection limit.

\section{References}

Burn, B.J.: 1966, MNRAS 133, 67

Donner, K.J. and Brandenburg, A.: 1990, $A \cup A$ 240, 289

Ehle, M. and Beck, R.: 1993, $A \& A$ (in press)

Gräve, R. and Beck, R.: 1988, $A \& A$ 192, 66

Hummel, E. and Gräve, R.: 1990, $A \& A$ 228, 315

Ishizuki, S., Kawabe, R., Ishiguro, M., Okumura, S.K., Morita, K.-I., Chikada, Y. and Kasuga, T.: 1990, Nature 344, 224

Krause, M., Hummel, E. and Beck, R.: 1989a, $A \& A$ 217, 4

Krause, M., Beck, R. and Hummel, E.: 1989b, $A \& A$ 217, 17

Manchester, R.N. and Mebold, U.: 1977, $A \& A$ 59, 401

Ruzmaikin, A.A., Shukurov, A.M. and Sokoloff, D.D.: 1988, Magnetic Fields of Galaxies, Kluwer: Dordrecht

Sokoloff, D., Shukurov, A. and Krause, M.: 1992, A\&A 264, 396

Turner, J.L. and Hurt, R.L.: 1992, $A p J$ 384, 72 